\title{
Scalable Multiplexed Drug-Combination Screening Platforms Using 3D Microtumor Model for Precision Medicine
}

\author{
Zhixiong Zhang, Yu-Chih Chen, Sumithra Urs, Lili Chen, Diane M. Simeone, \\ and Euisik Yoon*
}

Cancer heterogeneity is a notorious hallmark of this disease, and it is desirable to tailor effective treatments for each individual patient. Drug combinations have been widely accepted in cancer treatment for better therapeutic efficacy as compared to a single compound. However, experimental complexity and cost grow exponentially with more target compounds under investigation. The primary challenge remains to efficiently perform a large-scale drug combination screening using a small number of patient primary samples for testing. Here, a scalable, easy-to-use, high-throughput drug combination screening scheme is reported, which has the potential of screening all possible pairwise drug combinations for arbitrary number of drugs with multiple logarithmic mixing ratios. A "Christmas tree mixer" structure is introduced to generate a logarithmic concentration mixing ratio between drug pairs, providing a large drug concentration range for screening. A three-layer structure design and special inlets arrangement facilitate simple drug loading process. As a proof of concept, an 8-drug combination chip is implemented, which is capable of screening 172 different treatment conditions over 1032 3D cancer spheroids on a single chip. Using both cancer cell lines and patient-derived cancer cells, effective drug combination screening is demonstrated for precision medicine.

have identified thousands of mutations that are critical to cancer progression, $, 2,3]$ this genotypic method does not always pinpoint ideal cancer therapeutics due to the limited biological understanding. ${ }^{[4]}$ In addition, the presence of non-DNA genetic variations, including epigenetic modifications, lineage-specific changes and tumor microenvironment modulations, ${ }^{[5]}$ could make it even more complicated to correlate cancer cell genetic information with clinical consequences. ${ }^{[6,7]}$ These drawbacks can be addressed by empirical phenotypic drug testing, ${ }^{[8,9]}$ in which patient cancer cells are exposed to multiple treatments in vitro as a therapeutic guidance for individual patients. ${ }^{[10]}$

As compared to mono-drug treatment, drug combination has emerged as treatments for many diseases, ${ }^{[11]}$ especially for cancer due to its difficulty in treatment and cellular heterogeneity. ${ }^{[12]}$ Although many new cancer drugs have been developed, mono-drug treatments typically fail

\section{Introduction}

In recent decades, considerable efforts have been made in precision cancer therapy, which aims to customize appropriate treatment decisions based on individual cases. ${ }^{[1]}$ Though advances in DNA profiling and next-generation sequencing

\footnotetext{
Z. Zhang, Dr. Y-C. Chen, L. Chen, Prof. E. Yoon

Department of Electrical Engineering and Computer Science

University of Michigan

1301 Beal Avenue, Ann Arbor, MI 48109-2122, USA

E-mail: esyoon@umich.edu

Dr. Y.-C. Chen

University of Michigan Comprehensive Cancer Center

1500 E. Medical Center Drive, Ann Arbor, MI 48109, USA

S. Urs, Prof. D. M. Simeone

University of Michigan Health System

Ann Arbor, MI 48109, USA

Prof. E. Yoon

Department of Biomedical Engineering

University of Michigan

Ann Arbor, MI 48109, USA

The ORCID identification number(s) for the author(s) of this article can be found under https://doi.org/10.1002/smll.201703617.
}

DOI: 10.1002/smll.201703617 curing cancer ${ }^{[13]}$ due to the existence of alternative pathways to compensate the pathway target of drug. ${ }^{[14,15]}$ In order to overcome the limitations of mono-drug therapies, drug combinations, which aim to inhibit multiple redundant pathways of tumor cells, ${ }^{[16]}$ have been widely accepted for better therapeutic efficacy. ${ }^{[17]}$ To identify appropriate drug combinations, it is desirable to include more compound candidates, ${ }^{[18]}$ yet the resulting experimental complexity and cost increases exponentially. ${ }^{[19]}$ For example, investigation of 50 different compounds in pairwise combination yields ${ }_{50} C_{2}=1225$ different combinations. Furthermore, we assume 7 different concentration ratios for each combination, and 6 replicates for each treatment condition, which yields $1225 \times 7 \times 6 \times 10=51450$ experiments. This is not only costly but also time-consuming for a typical test panel of well-plate platforms.

In 2013, The US Food and Drug Administration expressed their dedication in developing novel combinatorial therapies, highlighting the need for innovative technologies to accelerate the discovery of novel drug combinations. ${ }^{[20,21]}$ To achieve highthroughput drug combination screening, several systems have been presented incorporating robotics and automatic handling. ${ }^{[22]}$ However, they are limited by complicated operation systems $^{[23]}$ and time-consuming serial processes. ${ }^{[21]}$ Microfluidics emerges as a promising technology for both clinical 
precision medicine and industrial-scale drug discovery, ${ }^{[24]}$ thanks to its capability of handling small samples and highly multiplexed operations for high-throughput assays. ${ }^{[25]}$ Previous microfluidic high-throughput drug screening platforms introduce a "Christmas tree structure" to generate a linear drug concentration gradient, but they are limited to the combination of two drugs, ${ }^{[26,27]}$ which may not meet the needs for highthroughput drug screening. In addition, since cells respond to different drug concentrations in a nonlinear manner, in some cases drug screening experiments require testing dosages ranging several orders of magnitude to calculate the $50 \%$ inhibition concentration (IC50). ${ }^{[28]}$ The narrow concentration range provided by conventional microfluidic linear gradient generators severely limits the use of microfluidics in drug screening. ${ }^{[29]}$ Previous work using microfluidic generating logarithmic concentration gradient requires complicated valve operation and interface system, which is not desirable for routine drug screening application. ${ }^{[30]}$

Furthermore, conventional in vitro cancer drug screening was mostly performed on 2D well-plates as a simple, fast, and cost-effective tool. ${ }^{[31]}$ However, accumulating evidence on in vitro cancer studies shows that a large number of cellular features and gene expression are skewed in a 2D culture environment, ${ }^{[32]}$ which makes it less reliable to make accurate clinical decisions. 3D cell culture systems have been widely used as better models in mimicking the in vivo tumor microenvironment, ${ }^{[3]}$ and have become increasingly popular in drug screening studies. ${ }^{[34]}$ In this work, we report a scalable, easy-to-handle, high-throughput drug combination screening scheme, incorporated with custom software for drug efficacy readout and data analysis. The presented microfluidic design enables screening of all possible pairwise drug combinations from arbitrary number of different drugs. As a proof of concept, we demonstrated an 8-drug screening chip with logarithmic concentration gradient, and performed drug combination screening experiment with multiple cell lines. Combining ${ }_{8} C_{2}=28$ drug combinations, 6 mixing ratios, and 6 replicates, plus some single compound control chambers, a total of 1032 drug efficacy screening experiments can be accomplished in a single 8-drug screening chip. Using the presented chip, we successfully performed drug combination screening of pancreatic cancer patient-derived cell lines, as a demonstration for precision medicine applications.

\section{Results and Discussion}

\subsection{Microfluidic Filter Structure for Cell Capture and Sphere Formation}

The proposed drug combination screening chip consists of 1032 microtumor culture units, $(28$ drug combinations $\times 7$ concentration mixing ratios +4 culture media controls) $\times 6$ replicates $=1032$. Cells loaded to the chip will be automatically distributed to all the culture chambers to form microtumors. To facilitate cell capturing and microtumor culturing, each culture unit is composed of a center sphere culture chamber, a ring chamber surrounding the center sphere culture chamber, and a thin gap ( $5 \mu \mathrm{m}$ in height, $50 \mu \mathrm{m}$ in length) connecting the two chambers. A total of 20 octagon micropillars $(5 \mu \mathrm{m}$ in height, $25 \mu \mathrm{m}$ in side length, and $25 \mu \mathrm{m}$ in spacing between pillars) are sparsely deployed between the polydimethylsiloxane (PDMS) thin gap to provide mechanical support and prevent the thin gap from collapsing (Figure 1a).

\subsection{Microfluidic Tree Structure as Logarithmic Concentration Gradient Generator}

We presented a "Christmas tree mixer" structure with nonuniform channels sizes to achieve a logarithmic mixing ratio gradient between two different compounds. The "Christmas tree mixer" is composed of five stages of microfluidic meander channels, with an incremental number from three to seven in each stage (Figure 2a). Solutions containing two different compounds are introduced from the top inlets and flow through the microchannel network. The fluid streams are combined in each branch channel stage, yielding mixture of distinct compositions, and splitting to the next stage. Finally, a concentration gradient is generated across the last stage of branching channels. ${ }^{[26]}$ The splitting ratio of the flow at each stage is determined by the flow resistance. According to Hagen-Poiseuille equation, hydraulic resistance of a channel is approximately inversely proportional to squared channel width. ${ }^{[35]}$ When all the microfluidic meander channels are designed to be of the same size (width, height, length), the flow resistance through all the branch channels are the same. In this case, a linear concentration gradient with an arithmetic progression ratio will be established in the last stage.

However, most drug screening experiments require log-scale concentration gradient covering a wider concentration range. In order to generate nonlinear concentration gradient, channels on both left and right sides are designed to be wider than those at the center, so that the flow resistance of meander channels on the sides are smaller than that of the channels at the center. For example, two drug compounds, A and B, are loaded on the two sides of the mixer (Figure 2a). Due to a small flow resistance in side channels compared to center channels, mostly of the flow will be guided side channels, while only a small portion mixed with each other at the center meander channels. In this case, when the mixture from the previous stage flowing to the next stage, a large volume of compound A from the channels on the left merged with the small volume of mixture compound $\mathrm{A}+\mathrm{B}$ from the center channel, yielding a mixture containing mostly A with a small portion of $\mathrm{B}$. The more drastically different the volumes are; the less amount of compound B is contained in the final mixture, the larger concentration ratio of compound $\mathrm{A}$ to compound $\mathrm{B}$ is. In this manner, we can generate a logarithmic concentration gradient (Figure 2b). (Quantitative analysis is described in the Supporting Information).

Another critical design of this chip is that meander channels at the last stage (i.e., fifth stage) of mixer array have the same dimension, instead of following the previously described rule of "side channels wider than center channels." This last stage meander channels are used as a "buffering layer" between the upstream "Christmas tree mixer" and downstream sphere culture chambers (Figure 2c). The rationale behind this last stage meander channel design is that the sphere culture chambers 

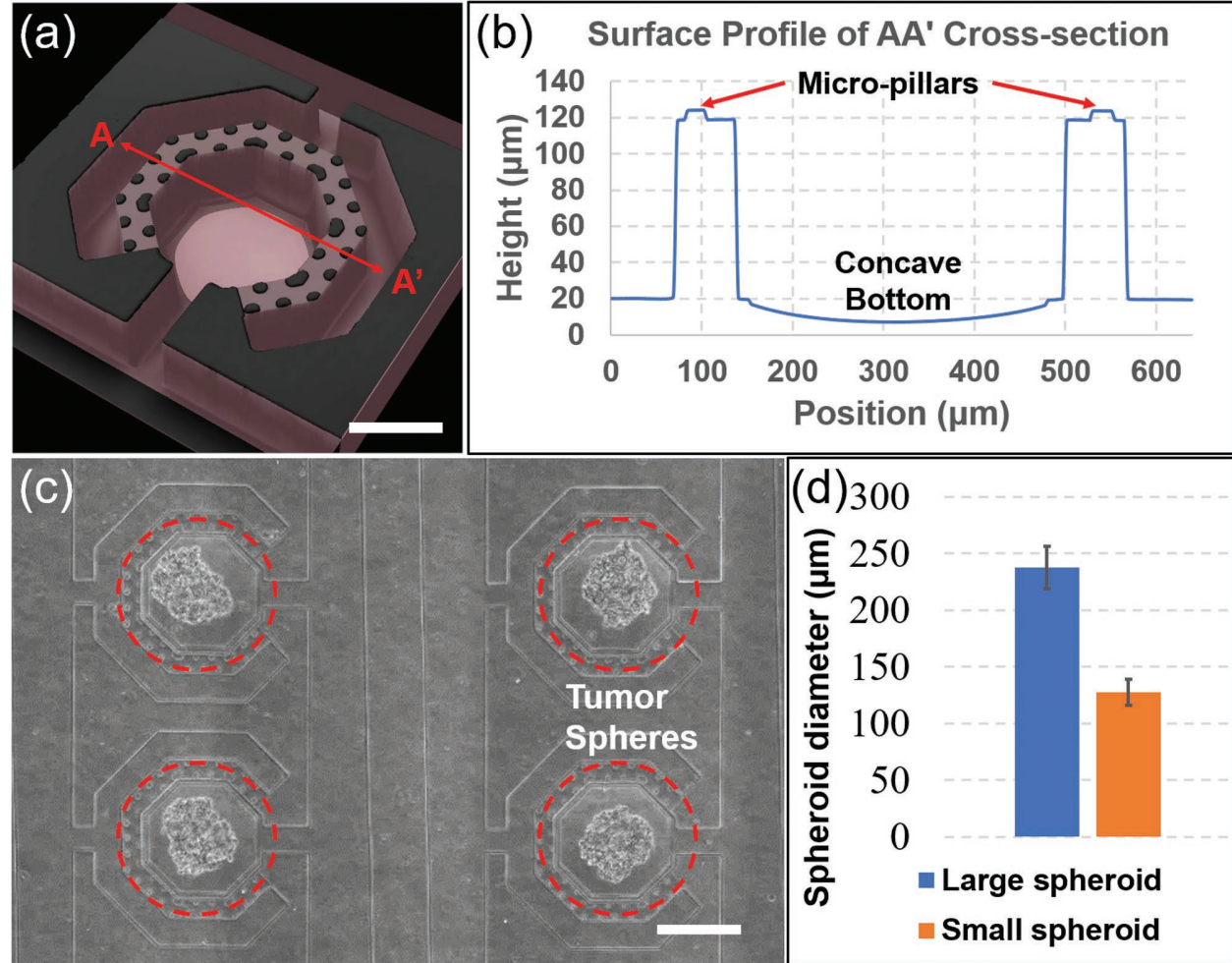

Figure 1. Spheroid formation in culture chambers. a) Laser confocal microscopy image of a unit chamber. Cells were captured at $5 \mu \mathrm{m}$ gap mechanically supported by micropillars. b) Spheroid culture chamber cross-section profile measured by Olympus OLS 4000 LEXT with a concave bottom of $16 \mu \mathrm{m}$ in depth. c) Microscopy image of SUM159 breast tumor spheroids forming in sphere culture chambers. (Scale bar $=200 \mu \mathrm{m}$ ) d) Size distribution of spheroids formed in the culture chambers.

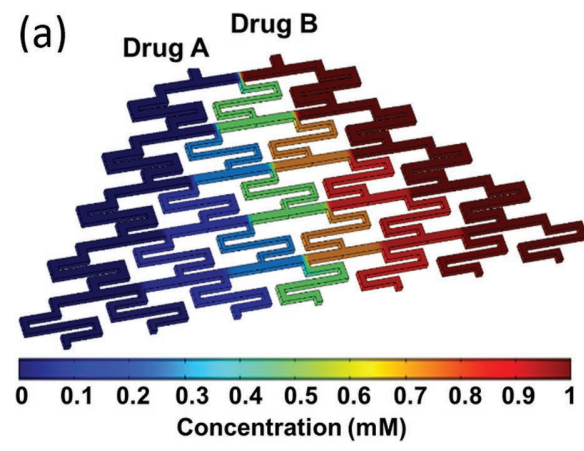

(b)

(c)

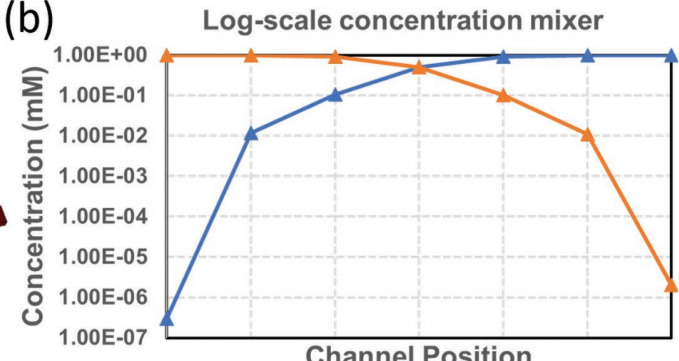

$\mp$ Drug A $\leftarrow$ Drug B

(d)

Flow Rate Distribution

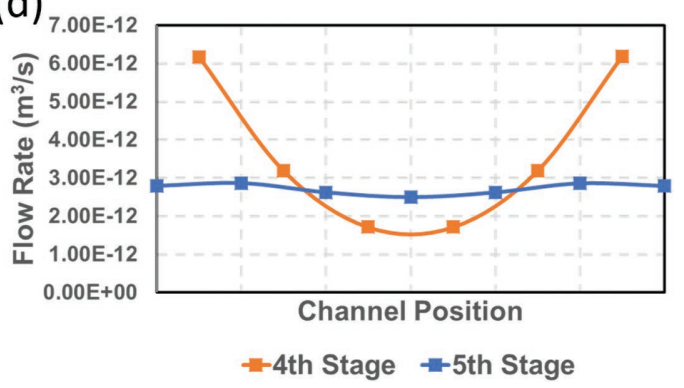

Figure 2. COMSOL simulation results for logarithmic "Christmas tree mixer" structure a) Concentration simulation of "Christmas tree mixer" using COMSOL. b) Measurement of the concentration of both drug A and drug B at the final stage of the mixer. c) Velocity simulation of "Christmas tree mixer" using COMSOL. d) Flow rate measurement of the channels in 4th stage and 5th stage (last stage), validating that the last stage can be a "buffering layer" to balance the flow rates among between branches. 
Table 1. Example drug inlets layout for 4 drugs. Number 1,2,3,4 stands for 4 different drugs.

\begin{tabular}{llll}
\hline 1 & 2 & 3 & 4 \\
2 & 4 & 1 & 3 \\
\hline
\end{tabular}

connected to the downstream also contributes to the equivalent resistance of the last stage of the "Christmas tree mixer." Even if the channel dimension of the last stage is carefully designed, because of the contributions of the hydraulic resistance from the downstream microfluidic structure, the equivalent resistance seen from the previous stage will be affected. On the other hand, the "Christmas tree mixer" also have an influence on the flow resistance of cell capture structures. When cells are loaded from cell inlets to cell culture chambers, the imbalanced channel design of "Christmas tree mixer" will also affect the cell loading uniformity, resulting in more cells loaded to chambers that are connected to side channels due to a lower flow resistance. Channels of the same dimension at the last stage of "Christmas tree mixer" help balance the cell loading and form tumor spheres of uniform size among all branches (Figure S2, Supporting Information).

We demonstrate the COMSOL simulation results of the presented mixer structure (Figure 2a), in which the channel dimension is designed to be symmetric. As described above, the channels at the last stage are the same in dimension (90 $\mu \mathrm{m}$ in width). To achieve the proper range of mixing ratios, the center channels are designed to be $60 \mu \mathrm{m}$ in width, the channels on both sides are $120 \mu \mathrm{m}$ in width, while the channels in between are $90 \mu \mathrm{m}$ in width. As a result, seven concentration ratios between drug $\mathrm{A}$ and drug $\mathrm{B}$ are achieved at a logarithmic gradient, ranging in $1: 10^{6}, 1: 100,1: 10,1: 1,10: 1$, $100: 1$, and $10^{6}: 1$ (Figure $2 \mathrm{~b}$ ), which is desirably wide for drug screening platforms.

\subsection{Mixer Array and Drug Inlets "Sudoku Puzzle"}

By deploying the "Christmas tree mixer" structures, we could generate a concentration gradient between two drugs. However, if all the ${ }_{8} C_{2}=28$ combinations of 8 drugs need to be screened using "Christmas tree mixer" side by side, at least $28 \times 2=56$ drug inlets should be deployed, which requires a very complicated microfluidic interface tubing system. To address this issue, adjacent Christmas tree mixers are designed to share a common drug inlet. However, this design requires special arrangement of the inlet array to guarantee the adjacent drug pairs covers all the possible combinations. For example, if we have 4 drugs, we could arrange the drug inlets as shown in Table 1.

In which, number 1-4 stand for 4 different drugs, and there is a "Christmas tree mixer" between each number pairs to generate concentration gradient. In this case, all the possible pairwise combinations, $1-2,2-3,3-4,1-3,2-4$, and $1-4$ are covered. However, the arrangement problem becomes much more complicated when the drug number becomes larger, for example, the computational complexity could reach $10^{26}$ possible permutations when the drug number comes to 16 . To make it possible for scaling-up, a general solution to the inlets arrangement is require so that the same design strategy could be applied to any arbitrary number of drugs. Although there are many possible solutions that could fulfill the requirements, we find a general solution for all even number of drugs. To make it convenient to explain, we rephrase the inlets arrangement problem to the following "Sudoku puzzle" problem:

Let $N$ be any even number, use number $1-N$ to fill in a table with $N$ columns and $N / 2$ rows. If we define the combination of horizontally adjacent numbers as a "pair," also define "1, 2" and " 2,1 " are the same pair. We will have $N \times(N-1) / 2$ pairs in the table. Try to fill in table, such that

a) Each row contains $N$ nonrepeating numbers from 1 to $N$.

b) All the $N \times(N-1) / 2$ pairs are nonrepeating, as well as covering all the possible combinations.

Since all the requirements are made on adjacency relationship, we find it very convenient to introduce an "adjacency relationship matrix" to keep record of the existing adjacent number. By following some rules in filling "adjacency relationship matrix" and putting certain numbers in the corresponding entries in "Sudoku puzzle," we are able to solve the "Sudoku puzzle" for any arbitrary even number (Supporting Information). As a demonstration, we showed the matrix for 16 drugs (Table 2).

In this work, due to the limitation of wafer area and availability of chemo-drugs, we only conduct experiment on devices for 4 and 8 drugs. However, we would like to show the potential of scaling up the design to allow combinatorial screening of arbitrary even number of drugs. As a proof of concept, we demonstrate a "sudoku puzzle" for 50 drugs, guaranteeing all the "horizontal adjacent pairs" are nonrepeating, while covering

Table 2. Example drug inlets layout for 16 drugs. Number $1-16$ stands for 16 different drugs.

\begin{tabular}{|c|c|c|c|c|c|c|c|c|c|c|c|c|c|c|c|}
\hline 1 & 2 & 3 & 4 & 5 & 6 & 7 & 8 & 9 & 10 & 11 & 12 & 13 & 14 & 15 & 16 \\
\hline 2 & 4 & 1 & 6 & 3 & 8 & 5 & 10 & 7 & 12 & 9 & 14 & 11 & 16 & 13 & 15 \\
\hline 3 & 1 & 5 & 2 & 7 & 4 & 9 & 6 & 11 & 8 & 13 & 10 & 15 & 12 & 16 & 14 \\
\hline 4 & 6 & 2 & 8 & 1 & 10 & 3 & 12 & 5 & 14 & 7 & 16 & 9 & 15 & 11 & 13 \\
\hline 5 & 3 & 7 & 1 & 9 & 2 & 11 & 4 & 13 & 6 & 15 & 8 & 16 & 10 & 14 & 12 \\
\hline 6 & 8 & 4 & 10 & 2 & 12 & 1 & 14 & 3 & 16 & 5 & 15 & 7 & 13 & 9 & 11 \\
\hline 7 & 5 & 9 & 3 & 11 & 1 & 13 & 2 & 15 & 4 & 16 & 6 & 14 & 8 & 12 & 10 \\
\hline 8 & 10 & 6 & 12 & 4 & 14 & 2 & 16 & 1 & 15 & 3 & 13 & 5 & 11 & 7 & 9 \\
\hline
\end{tabular}


all the possible pairwise combinations (Figure S5, Supporting Information).

\subsection{Multiple PDMS Layers for Drug Mixing and Routing}

As described in previous drug inlets array section, even if the inlet number could be reduced by sharing common drug inlets, there are still many inlets for each drug. For example, for a 16-drug combination screening chip, it is labor-intensive to load all the 16 drugs into $16^{2} / 2=128$ drug reservoirs, especially when there are many chips to operate. In order to further minimize the pipetting/tubing number, we present a three-layer chip design inspired by multilayer circuit board design. The three PDMS layers are routing layer, mixing layer, and lid layer from bottom to top (Figure 3a). In the mixing layer, different drug compounds are combined using previously mentioned "Christmas tree mixers" that are patterned on it, together with sphere culture chambers (Figure $3 b$ ). In the routing layer, a total of $N$ ( $N=$ number of drugs to be screened) microfluidic channels are implemented to connect multiple drug reservoirs for the same drug. Through these routing channels with extremely large cross-section area, thus negligible flow resistance compared to microchannels in the mixing layer, each drug compound can be automatically dispensed to all the drug inlets within seconds. The layout of the routing channels could be generated and optimized automatically using PADS Autorouter, a circuit board design software (Figure 3c). Since the flow resistance of the routing channels is extremely small

(a)

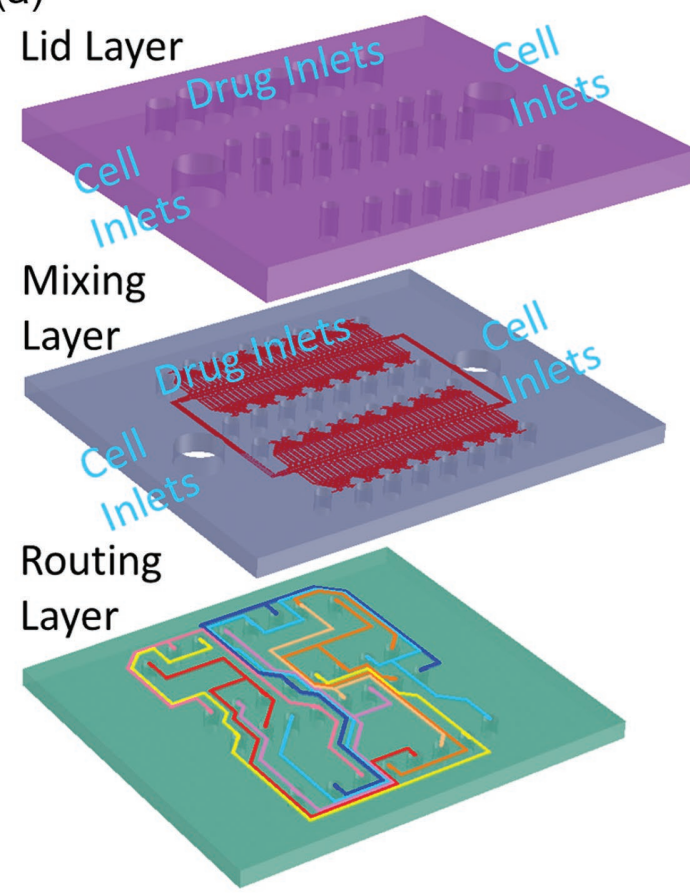

(around 4 orders of magnitude) compared to that of the mixing layer, the drug solution will fill all the drug reservoirs in seconds when one of the certain drug is loaded to any of the drug reservoirs. The lid layer PDMS covers the mixing layer to form an enclosed microfluidic system. The mixing PDMS layer was flipped to face upward before bonding to lid layer, so that cells could be captured at the $5 \mu \mathrm{m}$ thin gap in each individual chamber.

\subsection{Validation of Logarithmic Concentration Gradient On-Chip}

In order to test drug solution mixing on-chip, both fabricated linear concentration gradient device and logarithmic concentration gradient device were loaded with fluorescent dyes. The fluorescent intensity profile could be used to represent the concentration of different drugs. Phosphate-buffered saline (PBS no fluorescence) and three fluorescence dyes with different excitation wavelength were used: Tetramethylrhodamine (TRITC, red), Fluorescein (green), and DAPI (blue). After overlaying all the fluorescent images from each channel, we observed a rainbow-like color gradient formed in linear concentration gradient device (Figure 4a). By measuring the fluorescence intensity of all dyes, we verified each of the pairwise compound was mixed in six concentration ratios of 0\%:100\%, 20\%:80\%, 40\%:60\%, 60\%:40\%, 80\%:20\%, and $100 \%: 0 \%$, which is accordant with simulation results (Figure 4c). For logarithmic gradient generator, due to the tradeoff between the dynamic range and detection resolution
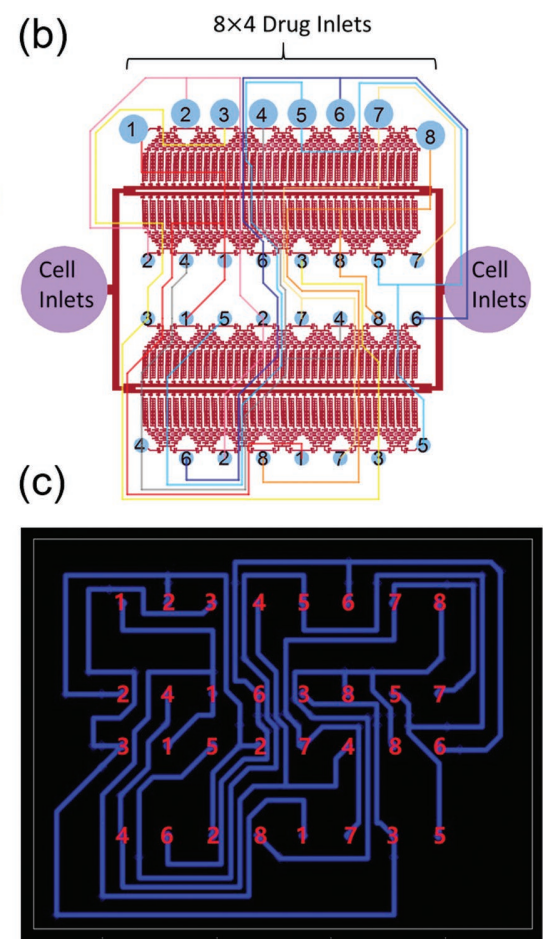

Figure 3. Schematics of multiplexed 8-drug combination screening chip. a) Separate views of three PDMS layers. 32 holes are punched through all the three PDMS layers for drug inlets using $6 \mathrm{~mm}$ biopsy punch, connecting the mixing and routing layers. b) Top view of the mixing layer: 32 inlets are allocated to 8 drugs, with a group of 4 connecting to each other with the same number in the routing layer. Before drug treatment, cells were loaded to the inlets and automatically deployed to 1032 culture chambers driven by gravity flow. Cell inlets are also used for drug outlet during drug treatment process. c) Top view of the routing layer, same number on different rows are connected in routing layer. 

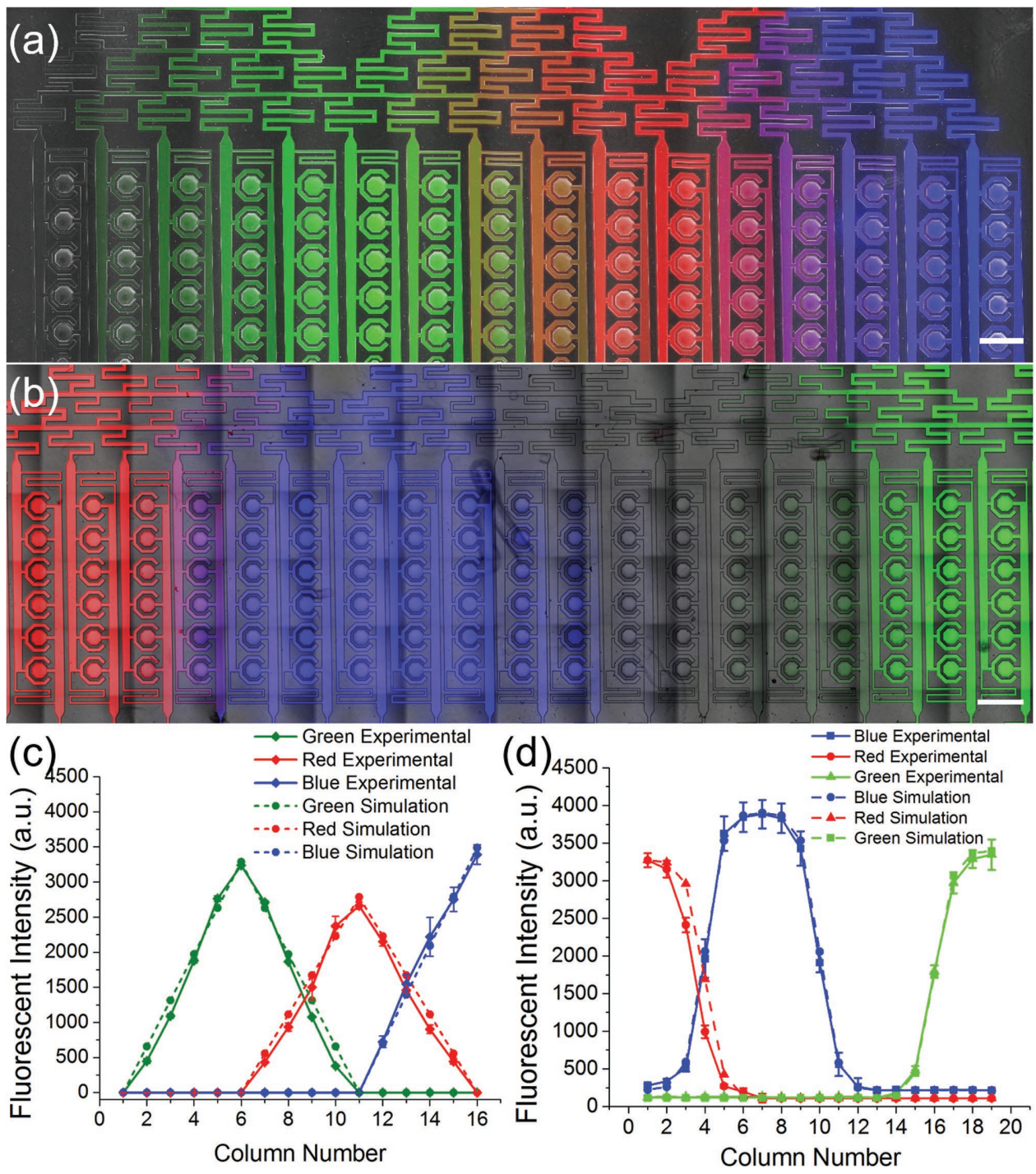

Figure 4. Generation of linear and logarithmic concentration gradient validated by fluorescent dye a) Linear gradient generation using PBS, Fluorescein (green), tetramethylrhodamine (red), and DAPI (blue), from left to right. The final image was created by overlapping images of the brightfield, FITC, TRITC, and DAPI channels. b) Logarithmic gradient generation using tetramethylrhodamine (red), DAPI (blue), PBS, and Fluorescein (green), from left to right. The final image was created by overlapping images of the brightfield, FITC, TRITC, and DAPI channel. c) Comparison of fluorescent intensity between linear gradient generation experiment (solid lines) and simulation results (dashed lines). d) Fluorescent intensity measurement of logarithmic gradient generation experiment (solid lines), which correlates well with simulation (dashed lines). (Scale bar $=500 \mu \mathrm{m}$ ).

of camera, different exposure times were needed to validation high concentration region and low concentration region, respectively. One image was taken with short exposure time $(30 \mathrm{~ms})$ to achieve larger detection range and avoid fluorescence saturation, so that the concentration relationship between $10 \%$, 50\%, and $90 \%$ could be verified (Figure $4 \mathrm{~b}$ ); While the other image is taken using long exposure time (300 ms), enabling the measurement of fluorescence intensity difference between $0 \%, 1 \%$ and $10 \%$ (Figure S6, Supporting Information). Thus, combining the measurement in these two images, it was validated that the logarithmic mixing ratio in fabricated device matches well with COMSOL simulation results (Figure $4 \mathrm{~d}$ ).

\subsection{Cancer Cell Loading and Sphere Formation}

For cell loading balance considerations, two cell inlets are used on both left side and right side. In the loading process, $500 \mu \mathrm{L}$ cell suspension is loaded to both cell loading inlets, while the 32 drug inlets are left empty, which are used as outlets. Driven by the pressure difference between cell loading inlets and drug loading inlets, cells are distributed to 1032 sphere culture chambers, and captured at the $5 \mu \mathrm{m}$ thin gap in each individual chamber. The chamber bottom was designed to be curved (Figure 1b), so that cells finally aggregate at the rounded bottom in central octagonal chamber, which is coated with Pluronic-108. After culturing cells for 
1 day, cells form spheroids of uniform size (Figure 1c). By regulating chamber dimensions, we are able to control the equivalent flow resistance of the cell culture gap. The larger the chamber dimension is, the smaller the flow resistance is. As a result, the more cells could be captured at the cell capture gap and the larger the spheroids could be finally formed. As a demonstration, we showed that two different sizes of spheres could be achieved (large sphere: $238 \pm 16 \mu \mathrm{m}$ in diameter, small spheres: $124 \pm 11 \mu \mathrm{m}$ in diameter), with two different chamber dimension designs (large chamber: $440 \mu \mathrm{m}$ in diagonal, small chamber: $240 \mu \mathrm{m}$ in diagonal), respectively (Figure $1 \mathrm{~d}$ ).

\subsection{Drug Combination Screening on Breast and Pancreatic Cancer Cell Lines}

To demonstrate the efficacy of high-throughput drug combination screening, we conducted a screening experiment with pancreatic cancer cell line, MIA PaCa-2, using a fabricated 8-drug logarithmic concentration gradient chip. All the pairwise combinations of seven chemo-drugs (Cisplatin, Docetaxel, Doxorubicin, Gemcitabine, Irinotecan, Oxaliplatin, Fluorouracil) together with culture media positive control were screened in a single-chip (Figure 5). As a result, we identified a few drug combinations with high synergistic indexes, which were highlighted

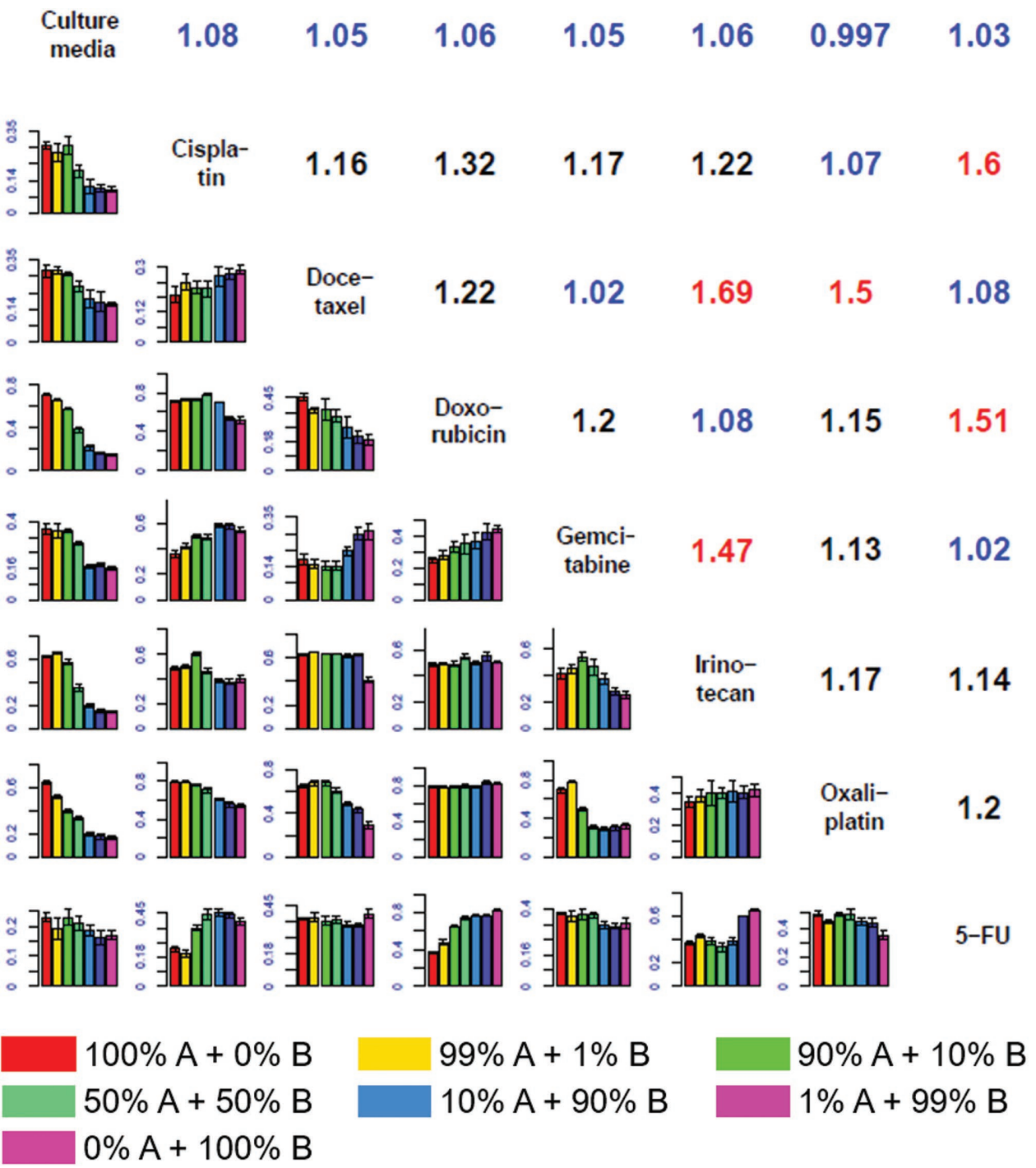

Figure 5. Drug combination screening results of control (culture media) and 7 drugs (Cisplatin, Docetaxel, Doxorubicin, Gemcitabine, Irinotecan, Oxaliplatin, 5-FU) using pancreatic cancer cell line MIA PaCa-2. Each subplot in the lower triangular table illustrates the cell death rate under the combination of drugs at each corresponding row and column. Each bar with different colors represent different concentration mixing ratio of certain drug combination. "Maximum Synergistic Index" (MSI) is denoted at upper triangle table, which is defined by the largest synergistic index among all the screened mixing ratio between the pair of drugs. Synergistic drug combination pairs with high MSI (>1.4) are highlighted in red, while those nonsynergistic ones with low MSI $(<1.1)$ are highlighted in blue. 
in red. For example, the combination of docetaxel + irinotecan, doxorubicin + 5-FU, docetaxel + oxaliplatin, and gemcitabine + Irinotecan showed synergistic indexes higher than 1.4, which have been proven to be more effective than single drugs in previous clinical and research literatures. ${ }^{[36-39]}$ While the nonsynergistic drug combinations, highlighted in blue in the table, such as cisplatin + oxaliplatin and gemcitabine $+5-\mathrm{FU}^{[40]}$ may result from their similar mechanism of action. Both cisplatin and oxaliplatin are DNA alkylating agents forming platinated intra-strand and inter-strand cross-link, interfering with DNA replication. ${ }^{[41]}$ While gemcitabine and 5 -FU are both belongs to nucleoside analog family of medication, which induces cell apoptosis by inhibiting the synthesis of new DNA. ${ }^{[42]}$ We further demonstrated the general usage for the presented chip for other types of cancer cell lines using SUM159 and MCF7, on a fabricated 4-drug screening chip with linear concentration gradient mixer (Figure S3, Supporting Information). We also showed reliable drug combination screening result among 4 fabricated 8-drug screening chips using SUM159 breast cancer cells, with negligible drug efficacy variations among different chips (Figure S4, Supporting Information).

\subsection{Drug Combination Screening on Pancreatic Cancer Patient- Derived Xenograft (PDX) Cell Line}

PDX models have been believed as an in vitro cancer model that is more physiologically relevant and readily applicable to the clinics, due to its preservation of the inter-tumor and intra-tumor heterogeneity, as well as the phenotypic and molecular characteristics of the original cancer. ${ }^{[43]}$ Thus, we further tested pancreatic cancer PDX cell lines to demonstrate the potential of presented chip for precision medicine. Combining the drug combination screening results of three PDX cell line samples, UM5, UM16, and UM53 (Figures S7-S9, Supporting Information), we established general drug responses of pancreatic cancer to a panel of combination treatments (Figure 6a). Among all drug combinations, a few of them with high average inhibition rate, such as fluorouracil + oxaliplatin, gemcitabine + oxaliplatin, and docetaxel + oxaliplatin, are identified as generally effective drug combinations for pancreatic cancer treatment. In fact, both fluorouracil + oxaliplatin and gemcitabine + oxaliplatin have been approved by US Food and Drug Administration (FDA) for pancreatic cancer combinatorial treatment. ${ }^{[4]}$ In addition, we also pinpointed the unique drug responses of each PDX sample. For UM16, none of the well-accepted drug combination was effective in inhibiting cell growth (Figure 6b). The nonconventional combination of gemcitabine and docetaxel, however, achieved a good drug efficacy. UM5 showed resistant to most of the drug compound except for the combination of $10 \%$ gemcitabine and $90 \%$ Oxaliplatin (Figure $6 \mathrm{c}$ ). The heterogeneous drug response among different patient samples confirmed the importance of customizing personalized chemotherapy for each individual. The successful combinatorial drug screening experiments using PDX cell lines suggest the potential of the presented platform in discovering new combination and precision medicine.

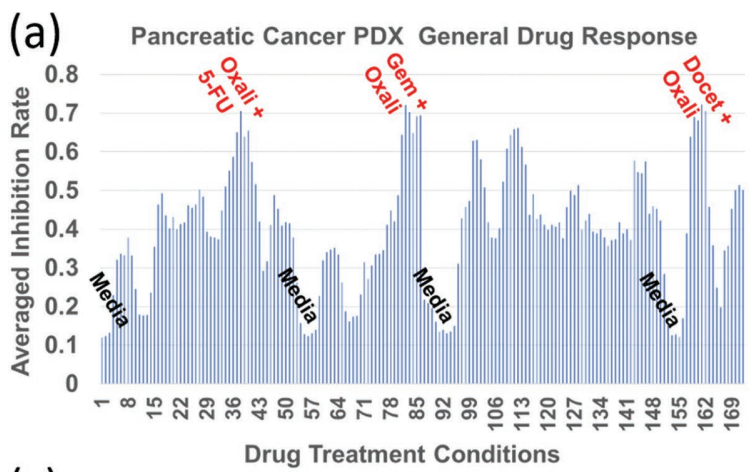

(C) UM5 Compared to General PDX Drug Response
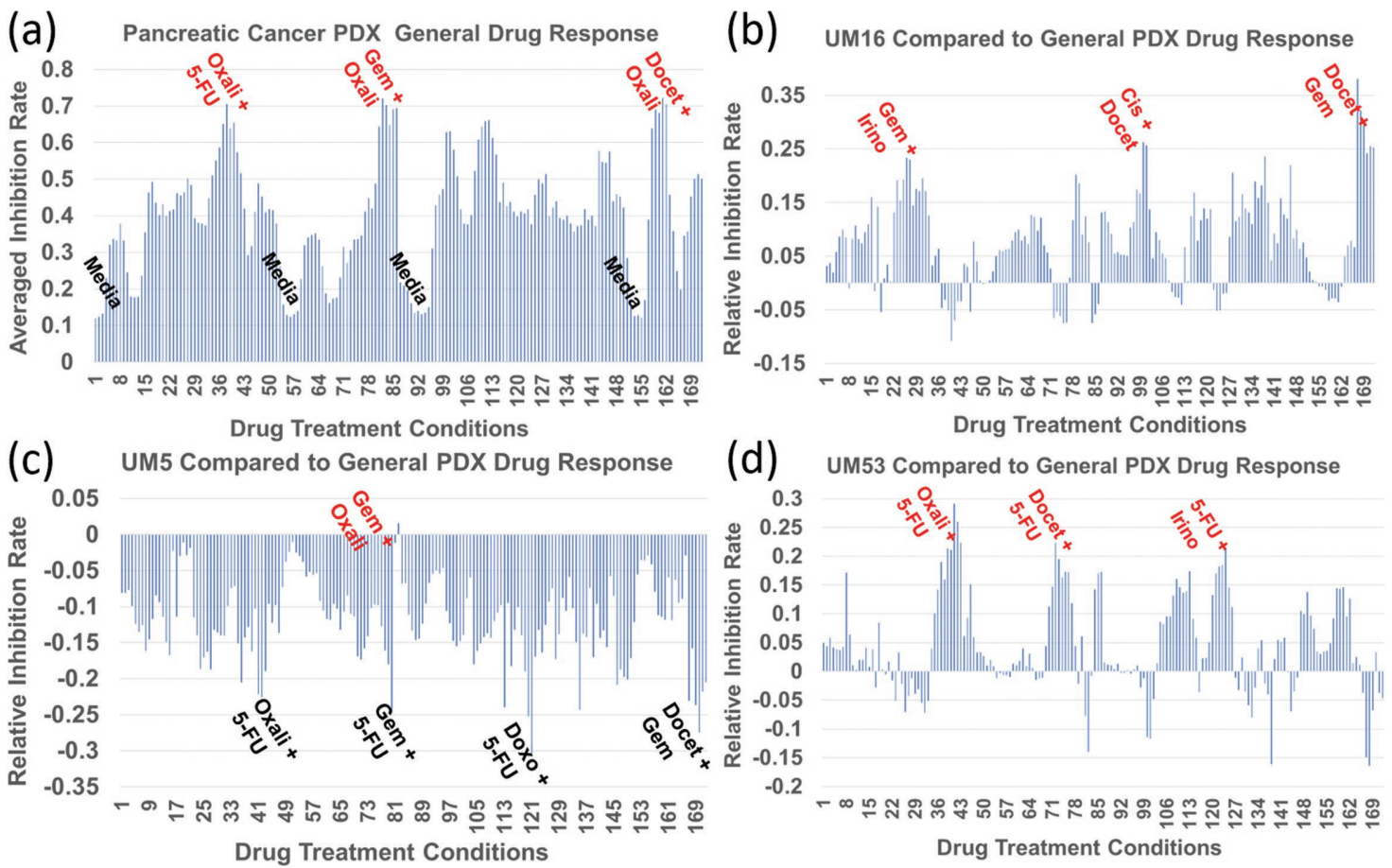

Figure 6. Drug combination screening results of control (culture media) and 7 drugs (Cisplatin, Docetaxel, Doxorubicin, Gemcitabine, Irinotecan, Oxaliplatin, 5-FU) using pancreatic cancer PDX cells. 172 different pairwise drug combinations with different mixing ratios are listed in X-axis. a) Average drug response of all PDX cells for 172 different drug treatment conditions. b) UM16 relative drug responses compared to the average drug response of all PDX cells. c) UM5 relative drug responses compared to the average drug response of all PDX cells. d) UM53 relative drug responses compared to the average drug response of all PDX cells. 


\section{Conclusion}

We reported a high-throughput, easy-handling, multiplex drug-combination screening platform scheme. By innovatively using three-layer PDMS structure, drug inlets sharing scheme, and specially arranged drug inlets array, we successfully demonstrated the feasibility of scaling up combinatorial drug screening for larger number of drugs. In addition, we presented a logarithmic concentration gradient generator, which provides a wider dynamic concentration range as compared to linear gradient generator. Furthermore, we adopted 3D tumor spheroids models for drug screening to better mimic in vivo tumor microenvironment. The design of spheroid culture chamber with $5 \mu \mathrm{m}$ cell capture gap, micropillar array, and rounded bottom structure guaranteed the formation of uniform spheroids of $238 \pm 16 \mu \mathrm{m}$ in size. As a demonstration of the design scheme, we fabricated an 8-drug combination screening chip, which generates ${ }_{8} C_{2}=28$ pairwise drug combinations, with 7 mixing ratios between each pair of drugs, yielding 172 different treatment conditions. Combining 6 replicates for each treatment condition, 1032 drug efficacy screening experiments can be accomplished in a single 8-drug screening chip. The drug screening experiment using pancreatic cancer cell line, MIAPaCa-2, identified the synergistic effects between docetaxel + Irinotecan, doxorubicin + 5-FU, docetaxel + oxaliplatin, and gemcitabine + Irinotecan, which match well with the clinical trial results reported in literature. We further verified the application of the fabricated chip in precision medicine using PDX cell lines, which better mimic the drug response of patients. We pinpointed the most synergistic drug combinations for each patient based on our screening results. The preliminary results verified the efficacy and synergistic effect of high-throughput drug combination screening. The presented design approach is easily scalable to incorporate a large number of drugs for largescale drug screening.

\section{Experimental Section}

Device Fabrication: The drug combination screening chip consisted of three PDMS layers: routing layer, mixing layer, and lid layer, from bottom to top (Figure 3a). The three PDMS layers were fabricated separately using standard soft lithography with silicon wafers as the mold. Four chrome-coated glass masks were used in fabricating the SU8 master molds. One of the four masks were used to create channels in routing layer $(800 \mu \mathrm{m}$ in width, $300 \mu \mathrm{m}$ in height), while the other three masks were used for mixing layer: the first mask was for thin cell capture gap (5 $\mu \mathrm{m}$ in height) with micropillars supporting structure; and the second mask defined patterns for the main microfluidic channels and sphere culture chambers (100 $\mu \mathrm{m}$ in height). The third mask was a dark-field mask with clear patterns of the central sphere culture chamber. The purpose of the third mask was to generate a rounded chamber bottom profile for better cell aggregation and sphere formation. SU8-2010 (MicroChem, NC9047158) was spun at $1000 \mathrm{rpm}$ for $30 \mathrm{~s}$ above the first two SU8 layers. Since the average SU8-2010 spreading thickness at $1000 \mathrm{rpm}$, which is around $20 \mu \mathrm{m}$, is thinner than the second SU8 layer $(100 \mu \mathrm{m})$, instead of covering all the surface, the newly spun SU8-2010 will form a rounded profile above the octagon sphere culture chamber patterns due to surface tension. The curvature is dependent on the viscosity of the photoresist, surface properties of the patterned surface, as well as the pattern dimensions. Using this protocol, a curved surface with center thickness of $16 \mu \mathrm{m}$ (Figure 1b) was being able to be created. PDMS (Dow Corning Sylgard 184, DC4019862) was cured on SU8 master at $100^{\circ} \mathrm{C}$ for 1 day and then peeled off from silicon mold; the three PDMS layers were treated with $\mathrm{O}_{2}$ plasma treatment at $100 \mathrm{~W}$ for $60 \mathrm{~s}$, followed by alignment and bonding together. $6 \mathrm{~mm}$ diameter holes were punched through the three PDMS layers as drug reservoirs, and $10 \mathrm{~mm}$ diameter holes as cell loading inlets. The fabricated chip was exposed to UV radiation to ensure sterilized conditions before use.

Cell Culture: Pancreatic cancer cell line and patient-derived cells were cultured both in bulk and in drug combination screening chip. Mia PACA-2, UM5, UM16, and UM53 cells were obtained from Dr. Simeone's Lab (University of Michigan, MI, USA). Pancreatic cells were cultured in RPMI (Gibco 11875) with 10\% FBS (Gibco 10082) and 1\% penicillin/ streptomycin (Gibco 15140). All the cells were cultured in polystyrene culture dishes at $37{ }^{\circ} \mathrm{C}$ and $5 \% \mathrm{CO}_{2}$, and passaged when cells reached over $80 \%$ confluency in the dish.

Cancer Drug Inventory: Cisplatin, cis-diamminedichloroplatinum (CAS 15663-27-1) was obtained from Cayman Chemical (Cat. No. 13119). Doxorubicin hydrochloride (CAS 25316-40-9) was obtained from Cayman Chemical (Cat. No. 15007). Gemcitabine (CAS 95058-81-4) was obtained from Accord (NDC No. 16729-092-03). Irinotecan hydrochloride (CAS 100286-90-6) was obtained from Cayman Chemical (Cat. No. 14180). Oxaliplatin (CAS 61825-94-3) was obtained from Cayman Chemical (Cat. No. 13106). Docetaxel (CAS 114977-28-5) was obtained from Cayman Chemical (Cat. No. 11637). Fluorouracil (CAS 51-21-8) was obtained from Sigma-Aldrich (Cat. No. F6627).

PDX Protocol: Patient-derived cells were obtained from PDX model in Dr. Simeone's Lab, following the protocol as in previous literature. ${ }^{[45]}$ Samples of human pancreatic adenocarcinomas were obtained within 30 min following surgical resection according to Institutional Review Board-approved guidelines. Tumors were suspended in and mechanically dissociated using scissors and then minced with a sterile scalpel blade over ice to yield $2 \times 2 \mathrm{~mm}$ pieces. The tumor pieces were washed with serum-free PBS before implantation. Eight-week-old male non-obese diabetic/severe combined immune deficiency (NOD/SCID) mice were anesthetized using an i.p. injection of $100 \mathrm{mg} \mathrm{kg}^{-1}$ ketamine and $5 \mathrm{mg} \mathrm{kg}^{-1}$ xylazine. A $5 \mathrm{~mm}$ incision was then made in the skin overlying the midabdomen, and three pieces of tumor were implanted s.c. The skin incision was closed with absorbable suture. The mice were monitored weekly for tumor growth for 16 weeks.

Before digestion with collagenase, xenograft tumors or primary human tumors were cut up into small pieces with scissors and then minced completely using sterile scalpel blades. To obtain single-cell suspensions, the resultant minced tumor pieces were mixed with ultrapure collagenase IV (Worthington Biochemicals, Freehold, NJ) in medium 199 (200 units of collagenase per $\mathrm{mL}$ ) and allowed to incubate at $37^{\circ} \mathrm{C}$ for 2.5 to $3 \mathrm{~h}$ for enzymatic dissociation. The specimens were further mechanically dissociated every 15 to 20 min by pipetting with a $10 \mathrm{~mL}$ pipette. At the end of the incubation, cells were filtered through a $40 \mathrm{Am}$ nylon mesh and washed with Hank's balanced salt solution (HBSS)/20\% fetal bovine serum (FBS) and then washed twice with HBSS.

Dissociated cells were counted and transferred to a $5 \mathrm{~mL}$ tube, washed twice with HBSS containing $2 \%$ heat-inactivated FBS, and resuspended in HBSS with $2 \% \mathrm{FBS}$ at concentration of $10^{6}$ per $100 \mu \mathrm{L}$. Sandoglobin solution $\left(1 \mathrm{mg} \mathrm{mL}^{-1}\right)$ was then added to the sample at a dilution of 1:20 and the sample was incubated on ice for $20 \mathrm{~min}$. The sample was then washed twice with HBSS/2\% FBS and resuspended in $\mathrm{HBSS} / 2 \%$ FBS. Antibodies were added and incubated for 20 min on ice, and the sample was washed twice with HBSS/2\% FBS. When needed, a secondary antibody was added by resuspending the cells in HBSS/2\%FBS followed by a 20-min incubation. After another washing, cells were resuspended in HBSS/2\% FBS containing 4',6-diamidino2-phenylindole (DAPI; $1 \mathrm{Ag} \mathrm{mL}^{-1}$ final concentration). The antibodies used were anti-CD44 allophycocyanin, anti-CD24 (phycoerythrin), and anti-H2K (PharMingen, Franklin Lakes, NJ) as well as anti-ESA-FITC (Biomeda, Foster City, CA), each at a dilution of 1:40. In all experiments using human xenograft tissue, infiltrating mouse cells were eliminated by discarding $\mathrm{H} 2 \mathrm{~K}$ (mouse histocompatibility class I) cells during flow 
cytometry. Dead cells were eliminated by using the viability dye DAPI Flow cytometry was done using a FACSAria (BD Immunocytometry Systems, Franklin Lakes, NJ). Side scatter and forward scatter profiles were used to eliminate cell doublets. Cells were routinely sorted twice, and the cells were reanalyzed for purity, which typically was $>97 \%$.

Sorted cells were washed with serum-free HBSS after flow cytometry and suspended in serum free-RPMI/Matrigel mixture (1:1 volume) followed by injection s.c. into the right and left midabdominal area using a 23-gauge needle. In separate experiments, mice were anesthetized with an i.p. injection of $100 \mathrm{mg} \mathrm{kg}^{-1}$ ketamine and $5 \mathrm{mg} \mathrm{kg}^{-1}$ xylazine, a median laparotomy was done, and either 1000 or 5000 sorted cells. Animals underwent autopsy at 28 days after cell implantation and tumor growth was accessed. Tissues were fixed in formaldehyde and examined histologically.

Cell Loading: To facilitate 3D cell culture, the device surface was coated with a well-characterized, commercially available, PEOterminated triblock polymer, Pluronic F108, which has been shown to be nonimmunogenic, nontoxic, and FDA approved for a wide usage in medical applications ${ }^{[46]}$ (Figure S1, Supporting Information). 5\% (w/w) Pluronic F-108 (CAS 9003-11-6, BASF) in DI water was loaded to the device $12 \mathrm{~h}$ before cell loading to create nonadherent surface. ${ }^{[47]}$ Before cell loading, devices were washed by PBS(Invitrogen, ILT10010023). For cell loading, $500 \mathrm{~mL}$ cell suspension was added to each of the cell loading inlets and was driven to all the cell culture chambers by gravity flow. After cell loading, cancer cells were allowed to aggregate to form spheroids and grow for $48 \mathrm{~h}$ in each sphere culture chambers after loaded to the chip. RPMI (Gibco 11875) with 10\% FBS (Gibco 10082) and $1 \%$ penicillin/streptomycin (Gibco 15140) was used as culture media to help cancer cells to form tight spheroid.

Drug Screening Protocol and Drug Efficacy Readout: After culturing for $48 \mathrm{~h}$, culture media were taken out from all the inlets. All the drugs were loaded to the corresponding inlets and replaced with new drug solution every $12 \mathrm{~h}$ before the hydraulic pressure between inlets and outlets fails to maintain the flow rate and mixing ratio. All the drugs were dissolved to targeted concentration in RPMI (Gibco 10082) serum free media. After $72 \mathrm{~h}$ treated with drug, the spheroid was stained with using Live/ Dead Viability/Cytotoxicity Kit for mammalian cells (Life technologies, L-3224), in which live cells were stained with green fluorescence and dead cells with red fluorescence, and were put in an incubator $\left(37^{\circ} \mathrm{C}\right.$, $5 \%\left(\mathrm{CO}_{2}\right)$ for $1 \mathrm{~h}$, followed by fluorescence microscopy imaging. The inhibition rate was calculated using the fluorescent intensity ratio of live cells to dead cells. A custom program was developed to analyze the inhibition rate and drug efficacy in a high-throughput manner (Figure S4b, Supporting Information).

Synergy Effect Analysis: To quantify the synergistic effect of various drug combinations, "Maximum Synergy Index" (MSI) was calculated inspired by loewe additivity. ${ }^{[48]}$ Based on dose equivalence principle, that for a given effect, dose $a$ of drug $\mathrm{A}$ is equivalent to dose $b_{a}$ of drug $\mathrm{B}$, and reciprocally. In addition, dose $b_{a}$ can be added to any other dose $b$ of drug $B$ to give the additive effect of the combination. The additive effect of drugs $A$ and $B$ can be expressed as ${ }^{[49]}$

$\operatorname{Effect}(a+b)=E_{\mathrm{A}}\left(a+a_{b}\right)=E_{\mathrm{B}}\left(b_{a}+b\right)$

To quantify the combination synergistic index, the linear interpolation value of each combination with different mixing ratio was first calculated based on single drug effect. For example, drug A with dose $a$ yields $I_{a} \%$ inhibition rate, while drug $B$ with dose $b$ yields $I_{b} \%$ inhibition rate. The combination of drug $A$ and drug $B$ with the mixing ratio of $1: 1$ should yields $I_{a b}=\left(I_{a} \%+I_{b} \%\right) / 2$ inhibition rate. The "synergy index" of drug $A$ and drug $B$ with certain mixing ratio is defined as dividing the experimental inhibition rate $I_{a b, \exp }$ by theoretical inhibition rate $I_{\text {ab,theory }}$ The largest synergistic index among all the screened mixing ratio between drug $A$ and drug $B$, which has the best chance of achieving the best therapeutic result, is defined as the MSI of the drug combination between $\mathrm{A}$ and $\mathrm{B}$. A MSI greater than 1 indicates a synergistic drug combination, since the maximum inhibition rate of the drug combination is higher than the linear interpolation of individual drugs.
Image Acquisition: The microfluidic chips were imaged using an inverted microscope (Nikon). The bright-field and fluorescent images were taken with a 10x objectives and a charge-coupled device camera (Coolsnap HQ2, Photometrics). FITC and TRITC filter sets were used for the fluorescent imaging. The microfluidic cell chamber array was scanned with a motorized stage (ProScan II, Prior Scientific). Before each scanning, the stage was leveled to ensure the image remained in the focus throughout the whole imaging area. To ensure optimized image quality, autofocusing was done after imaging every 4 frames. After scanning, the Nikon NIS-Elements Basic Research software module was used to stitch individual images into a large image for analysis. The fluorescent intensity of each spheroid was quantified using Nikon Research Basics software. In order to cancel the interference from background noise and substrate absorption, background fluorescent intensity was subtracted from the signal.

COMSOL Simulation: The COMSOL simulation in this work used "laminar flow" model for fluid dynamics. Boundary conditions were set as follows: inlet pressure $=100 \mathrm{~Pa}$, outlet pressure $=0 \mathrm{~Pa}$, diffusion coefficient $=2 \times 10^{-9} \mathrm{~s}^{-1}$, which equals to the diffusion coefficient of cisplatin dissolved in liquid solvent. ${ }^{[50]}$ In addition, "3D transport of diluted species" was applied to simulate the mixing between two chemical compounds, in which the model parameters were set as follow: $T=293.15 \mathrm{~K}$, fluid density $=1000 \mathrm{~m}^{-3}$, dynamic viscosity = $0.00103 \mathrm{~Pa} \cdot \mathrm{s}$ The concentration of the compound loaded to two inlets are set as: inflow $1=0 \mathrm{mmol} \mathrm{m}^{-3}$, inflow $2=1 \mathrm{mmol} \mathrm{m}^{-3}$. Normal physics-controlled mesh was used.

Data Analysis and Processing: Spheroids smaller than $30 \mu \mathrm{m}$ in diameter were not included for analysis. Statistical analyses and synergy index table plot were performed using $\mathrm{R}$ (version 3.3.2). Two-tailed, unpaired Student's $t$-tests were used for all comparisons and significance level of $p<0.05$ was used to consider statistical significance. * refers to $p<0.05$, ** refers to $p<0.01$, and *** refers to $p<0.001$. Results are presented as mean $\pm \mathrm{SD}$.

\section{Supporting Information}

Supporting Information is available from the Wiley Online Library or from the author.

\section{Acknowledgements}

This work was supported in part by the National Institutes of Health (1R21CA17585701, 1R21CA195016). Y.-C.C. acknowledges the support from Forbes Institute for Cancer Discovery. The Lurie Nanofabrication Facility of the University of Michigan (Ann Arbor, MI) are greatly appreciated for device fabrication.

\section{Conflict of Interest}

The authors declare no conflict of interest.

\section{Keywords}

3D spheroid, drug combination, high-throughput drug screening, microfluidics, precision medicine

Received: October 16, 2017

Revised: April 12, 2018

Published online: September 21, 2018

[1] D. Gonzalez de Castro, P. A. Clarke, B. Al-Lazikani, P. Workman, Clin. Pharmacol. Ther. 2013, 93, 252.

[2] J. Gagan, E. M. Van Allen, Genome Med. 2015, 7, 80. 
[3] C. Cummings, E. Peters, L. Lacroix, F. Andre, M. Lackner, Clin. Transl. Sci. 2016, 9, 283.

[4] M. Arnedos, C. Vicier, S. Loi, C. Lefebvre, S. Michiels, H. Bonnefoi, F. Andre, Nat. Rev. Clin. Oncol. 2015, 12, 693.

[5] Y. J. Park, R. Claus, D. Weichenhan, C. Plass, Epigenet. Dis. 2010, 25.

[6] B. Lehner, Nat. Rev. Genet. 2013, 14, 168.

[7] M. Schneider, J. Schüler, R. Höfflin, N. Korzeniewski, C. Grüllich, W. Roth, D. Teber, B. Hadaschik, S. Pahernik, M. Hohenfellner, S. Duensing, Urol. Oncol.: Semin. Orig. Invest. 2014, 32, 877.

[8] W. Zheng, N. Thorne, J. McKew, Drug Discovery Today 2013, 18, 1067.

[9] J. Moffat, J. Rudolph, D. Bailey, Nat. Rev. Drug Discovery 2014, 13, 588.

[10] E. W. Esch, A. Bahinski, D. Huh, Nat. Rev. Drug Discovery 2015, 14, 248.

[11] B. Al-Lazikani, U. Banerji, P. Workman, Nat. Biotechnol. 2012, 30, 679.

[12] R. Fisher, L. Pusztai, C. Swanton, Br. J. Cancer 2013, 108, 479.

[13] K. Masui, B. Gini, J. Wykosky, C. Zanca, P. Mischel, F. Furnari, W. Cavenee, Carcinogenesis 2013, 34, 725.

[14] G. R. Zimmermann, J. Lehár, C. T. Keith, Drug Discovery Today 2007, 12, 34.

[15] C. Holohan, S. Van Schaeybroeck, D. B. Longley, P. G. Johnston, Nat. Rev. Cancer 2013, 13, 714.

[16] V. T. Devita, R. C. Young, G. P. Canellos, Cancer 1975, 35, 98.

[17] D. A. Yardley, Int. J. Breast Cancer 2013, 2013, 1.

[18] R. Macarron, M. N. Banks, D. Bojanic, G. S. Sittampalam, Nat. Rev. Drug Discovery 2011, 10, 188.

[19] J. Tang, L. Karhinen, T. Xu, A. Szwajda, B. Yadav, K. Wennerberg, T. Aittokallio, PLoS Comput. Biol. 2013, 9, e1003226.

[20] US Food and Drug Administration, Guidance for Industry: Codevelopment of Two or More New Investigational Drugs for Use in Combination, 2013, 1.

[21] L. A. M. Griner, R. Guha, P. Shinn, R. M. Young, J. M. Keller, D. Liu, I. S. Goldlust, A. Yasgar, C. Mcknight, M. B. Boxer, D. Y. Duveau, J.-K. Jiang, S. Michael, T. Mierzwa, W. Huang, M. J. Walsh, B. T. Mott, P. Patel, W. Leister, D. J. Maloney, C. A. Leclair, G. Rai, A. Jadhav, B. D. Peyser, C. P. Austin, S. E. Martin, A. Simeonov, M. Ferrer, L. M. Staudt, C. J. Thomas, Proc. Natl. Acad. Sci. USA 2014, 111, 2349.

[22] S. Michael, D. Auld, C. Klumpp-Thomas, A. Jadhav, W. Zheng, N. Thorne, C. Austin, J. Inglese, A. Simeonov, Assay Drug Dev. Technol. 2008, 6, 637.

[23] G.-S. Du, J.-Z. Pan, S.-P. Zhao, Y. Zhu, J. M. D. Toonder, Q. Fang, Anal. Chem. 2013, 85, 6740.

[24] R. Riahi, A. Tamayol, S. A. M. Shaegh, A. M. Ghaemmaghami, M. R. Dokmeci, A. Khademhosseini, Curr. Opin. Chem. Eng. 2015, 7, 101.

[25] Y.-C. Chen, Z. Zhang, S. Fouladdel, Y. Deol, P. N. Ingram, S. P. Mcdermott, E. Azizi, M. S. Wicha, E. Yoon, Lab Chip 2016, 16, 2935 .
[26] J. Kim, D. Taylor, N. Agrawal, H. Wang, H. Kim, A. Han, K. Rege, A. Jayaraman, Lab Chip 2012, 12, 1813.

[27] D. An, K. Kim, J. Kim, Biomol. Ther. 2014, 22, 355.

[28] R. Tallarida, L. S. Jacob, Dose-Response Relation in Pharmacology, Springer New York, New York 2012.

[29] B. G. Chung, L. A. Flanagan, S. W. Rhee, P. H. Schwartz, A. P. Lee, E. S. Monuki, N. L. Jeon, Lab Chip 2005, 5, 401.

[30] J. Y. Yun, S. Jambovane, S.-K. Kim, S.-H. Cho, E. C. Duin, J. W. Hong, Anal. Chem. 2011, 83, 6148.

[31] J. H. Tsui, W. Lee, S. H. Pun, J. Kim, D.-H. Kim, Adv. Drug Delivery Rev. 2013, 65, 1575.

[32] D. Antoni, H. Burckel, E. Josset, G. Noel, Int. J. Mol. Sci. 2015, 16, 5517.

[33] A. Nyga, U. Cheema, M. Loizidou, J. Cell Commun. Signaling 2011, 5, 239.

[34] Z. Zhang, Y.-C. Chen, Y.-H. Cheng, Y. Luan, E. Yoon, Lab Chip 2016, 16, 2504.

[35] N. A. Mortensen, F. Okkels, H. Bruus, Phys. Rev. E 2005, 71, 057301.

[36] B. Burtness, L. Thomas, R. Sipples, M. Mcgurk, S. Salikooti, M. Christoforou, G. Mirto, R. Salem, J. Sosa, R. Kloss, Z. Rahman, G. Chung, J. Lacy, J. R. Murren, Cancer J. 2007, 13, 257.

[37] M. W. Oster, R. Gray, L. Panasci, M. C. Perry, Cancer 1986, 57, 29.

[38] T. J. Ettrich, L. Perkhofer, G. V. Wichert, T. M. Gress, P. Michl, H. F. Hebart, P. Büchner-Steudel, M. Geissler, R. Muche, B. Danner, V. Kächele, A. W. Berger, M. Güthle, T. Seufferlein, BMC Cancer 2016, 16, 21.

[39] A. Lipton, C. Campbell-Baird, L. Witters, H. Harvey, S. Ali, J. Clin. Gastroenterol. 2010, 44, 286

[40] G. Bellone, A. Carbone, V. Busso, T. Scirelli, A. Buffolino, C. Smirne, A. Novarino, O. Bertetto, L. Tosetti, G. Emanuelli, Cancer Biol. Ther. 2006, 5, 1294

[41] R. K. Mehmood, Oncol. Rev. 2014, 8, 97.

[42] Y. F. Hui, J. Reitz, Am. J. Health-Syst. Pharm. 1997, 54, 162.

[43] S. Y. C. Choi, D. Lin, P. W. Gout, C. C. Collins, Y. Xu, Y. Wang, Adv. Drug Delivery Rev. 2014, 79-80, 222.

[44] https://www.fda.gov/, Center for drug evaluation and research, application number: 207793 Origls000.

[45] C. Li, D. G. Heidt, P. Dalerba, C. F. Burant, L. Zhang, V. Adsay, M. Wicha, M. F. Clarke, D. M. Simeone, Cancer Res. 2007, 67, 1030.

[46] V. A. Liu, W. E. Jastromb, S. N. Bhatia, J. Biomed. Mater. Res. 2002, 60: 126.

[47] Y.-C. Chen, X. Lou, Z. Zhang, P. Ingram, E. Yoon, Sci. Rep. 2015, 5, 12175.

[48] J. C. Boik, R. A. Newman, R. J. Boik, Stat. Med. 2008, 27, 1040.

[49] J. Foucquier, M. Guedj, Pharmacol. Res. Perspect. 2015, 3, e00149.

[50] T. Panczyk, A. Jagusiak, G. Pastorin, W. H. Ang, J. Narkiewicz-Michalek, J. Phys. Chem. C 2013, 117, 17327.

[51] K. A. Lipinski, L. J. Barber, M. N. Davies, M. Ashenden, A. Sottoriva, M. Gerlinger, Trends Cancer 2016, 2, 49.

[52] R. H. Shoemaker, Nat. Rev. Cancer 2006, 6, 813. 